\title{
FEATURES OF CRITICAL FACILITIES DETERMINING FOR THE FUEL AND ENERGY COMPLEX IN RESEARCH OF FUEL AND ENERGY SUPPLY
}

\author{
Beresneva N.M.: , Pyatkova N.I. \\ Melentiev Energy Systems Institute (ESI) SB RAS, Irkutsk, Russia
}

\begin{abstract}
The article reveals the features of selecting critical facilities for the fuel and energy complex (FEC CF) within joint industry and general energy model research. A comparative assessment of the methodology for determining the list of critical industry objects and the methodology for determining the list of FEC CF is given in this paper. A scheme for the preparation and analysis of the fuel and energy complex calculated states is described, the analyzed most important and related model indicators are highlighted, an integral indicator for assessing the criticality of elements is proposed. Also is provided a formalized description of the fuel and energy functioning optimization model and reveals a three-stage scheme for working with it in determining in the CF of FEC based on industry critical elements.
\end{abstract}

\section{Introduction}

The task of critical facilities(CF) identifying for the fuel and energy complex (FEC) his-torically appeared as a evolvement of the identifying industry critical elements task. The relevance of this task was justified by the need to assess these elements criticality degree in the conditions of energy systems joint functioning, taking into account reservation and the interchangeability of resources. The both problems solution was based on the use of a model and instrumental apparatus reflecting the technological and territorial structure of the research objects (sectoral systems and fuel and energy complex). Moreover, the task of the $\mathrm{CF}$ finding for the fuel and energy complex gave the evolvement of the FEC functioning model for in terms of detailing sectoral schemes (namely the gas industry, as it is for this system the methodology for determining industry critical elements was previously developed and tested) and in terms of modeling the reserve capabilities in electrical and heat power sys-tem at the technological level of their presentation in the model. At the methodological level the ideas of assessing the vulnerability of critical infrastructures' elements [1-2], to which relate the fuel and en-ergy complex and its sectoral systems [3], were used and developed, and schemes for identifying critical elements of the gas industry [4-5].

\section{Features of the choice of CF for FEC}

The methodology for determining the CFP for the fuel and energy complex is based on scheme of stage-bystage multilevel model studies (fig. 1). This fact made it possible to assert that:
- the significance of the same critical element for the industry and for the fuel and energy complex may be different, since when determining the CF of the fuel and energy complex, the systemic effect of interaction between energy industries is taken into account;

- the list of sectoral critical elements can be considered as potential CF of the fuel and energy complex, can be used to set calculated conditions in model studies of the fuel and energy complex;

- the list of the CF for the fuel and energy complex may not correspond to the list of sectoral critical elements, it will include sectoral elements that are really critical in the conditions of the interconnected work of the industries.

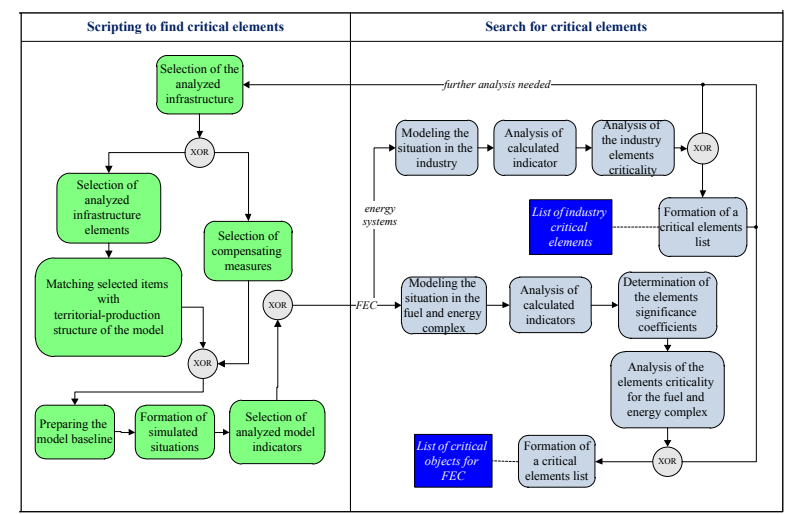

Fig. 1 Scheme for the selection of CF for the fuel and energy complex based on industry critical elements.

This methodology is similar to the methodology for selecting industry critical elements in terms of the formation and assessment of hypothetical calculated states, in terms of using for these purposes the relative deficits of energy resources in various territories,

\footnotetext{
Corresponding author: beresneva $@$,isem.irk.ru
} 
followed by obtaining quantitative and expert assessments of the criticality these elements. The systemic-functional complexity of the fuel and energy complex is expressed in the presence of energy resources multitude, the processes of their processing and transformation, in the possibility of their interchangeability at electric power system and heat power. This requires taking into account the resulting deficits for all resources using the possibilities of structural redundancy. Comparative analysis of methods for determining critical elements at the sectoral and general energy levels showed the following results.

1. Both methods are focused on the analysis of calculated states formed for the scenarios of analyzed elements outages. Thus, the inoperability consequences of these elements are assessed. When determining sectoral critical elements, it is also possible to assess the consequences of these elements inoperability when compensating measures are introduced. This mechanism [4] is used to adjust the list of sectoral critical elements, allows to exclude elements whose inoperability can be largely compensated for by connecting the reserve capabilities of the industry.

2. Both methods involve model calculations with a different number of simultaneously disconnected elements (in set theory, the size of a set is called its cardinality). When determining the sectoral critical elements, the calculations are analyzed separately for single or multiple disconnections of elements [4]. The methodology for determining the CF for the fuel and energy complex provides for the possibility of joint analysis of calculations with disconnections of groups of elements of different power.

3. Both methods include the idea of assessing the criticality of elements from the standpoint of changing the degree of energy supply to consumers. Therefore, the analysis in both cases involves the relative deficits of energy resources by territories, or by each sectoral system as a whole. In the fuel and energy complex model, which includes more than one industry, this assessment has complex, taking into account the total relative deficits of various energy resources for different categories of users. It also foreseen an assessment of the importance of the analyzed model indicators.

4. A large number of analyzed states required the elimination of states with an acceptable level of energy deficit in the system as a whole. In the case of general energy studies, this provision applies to each sectoral system. The level of permissible relative deficits is established by experts.

\section{Fuel and energy complex research models for analyzing the reliability of fuel and energy supply}

Complex scenario calculations in determining the fuel and energy complex $\mathrm{CF}$ are carried out on the basis of fuel and energy complex functioning models. These models include the main branch blocks that simulate the interconnected work of branch objects in varying degrees of territorial and technological detail. In the temporal aspect, they are focused on the daily aspect, since at longer time intervals, the consequences of a failure in the operation of industry facilities for the fuel and energy complex as a whole can be leveled. Research models are represented by a triplet $M(Z, S, I)$, where $Z=\left\{z_{t}\right\}, t=\overline{1, T}$ - scenarios corresponding to hypothetical states of the fuel and energy complex; $S$ - territorial-production structure of the fuel and energy complex and its branch systems; $I$ - information base of the model.

The structure of the research model is represented by a set $S(R E S, R E G, O B J)$, where $R E S=\left\{R E S_{i}\right\}, i=\overline{1, I}$ energy resources adopted in the model, $R E G=\left\{\operatorname{reg}_{w}\right\}$, $w=\overline{1, W}$ - territorial units of the model, $O B J\left(O B J^{\text {tech }}, O B J^{e c}\right)$ - objects of the model corresponding to energy or to economic-organizational structures of FEC. In each such object, set of processes $\left\{p_{n}\right\}$ are implemented:

$O B J_{v}=\left\{p_{n}\right\}$,

$v=\overline{1, V}, n=\overline{1, N}$, where

$v$ - object index,

$N$ - the number of processes implemented in the object.

The processes themselves are represented by a multitude

$P=\left\{p_{q}\right\}, q=\overline{1, Q}$, including technologies such as:

- resources extraction (or production);

- resources processing and transformation;

- resources diversification;

- resources storage;

- resources transportation;

- resource consumption.

The objects of the model are tied to its territorial units and are part of the industry systems included in the fuel and energy complex:

$F E C=\left\{E S_{f}\right\}, f=\overline{1, F}$

$E S_{f}=\left\{O \operatorname{Obj}_{g}\right\}, g=\overline{1, G}$, where

$F E C$ - fuel and energy complex of the country,

$E S_{f}$ - industry system ( $F$ - number of sectors),

$O b j_{g}$ - set objects of the industry system $f$.

The information base of the model $I(D I, D R)$ is represented by the initial $D I$ and calculated $D R$ information. Initial information $D I\left(I^{i n p} . I^{\text {rat }}\right)$ includes subject $I^{\text {inp }}$ (statistical, regulatory-reference) and prepared for calculation (converted subject) $I^{\text {rat }}$ model data, where

$I^{i n p}=\left\{i_{j}^{i n p}\right\}, j=\overline{1, J}$ - elements of initial information,

$I^{\text {rat }}=\left\{i_{k}^{r a t}\right\}, k=\overline{1, K}$ - model elements (its variables, coefficients),

$F=\left\{F_{k}^{j}: i_{j}^{i n p} \rightarrow i_{k}^{r a t}\right\}$ - algorithms for preparing model data based on subject information.

Calculated information $R=\left\{i_{l}^{o p t}\right\}, l=\overline{1, L}$ ( $L$ is the number of model variables) - the result of transforming model information $\left\{i_{l}^{\text {rat }}\right\}$ by an external solver. In the course of analysis, calculated information can be aggregated by groups of territorial units.

Technically, work with the fuel and energy complex models is represented by the processes of preparation and verification of data, procedures for the formation, 
calculation and analysis of various options for the fuel and energy complex functioning. The starting point of research is the annual balanced variant of the model, on the basis of which daily variant are subsequently formed (the variant of the most loaded day, variants for disconnecting fuel and energy facilities). Such a chain of work is founded by the composition and level of presentation of statistical and analytical industry information, the relative ease of balancing annual indicators Corrective coefficients used to obtain daily variants are formed expertly based on the specifics of the analyzed situations, or are calculated in the presence of monthly industry reports.

Thus, the scheme of work with the fuel and energy complex model is conceptually represented by three stages:

- the stage of debugging a balanced annual variant;

- the stage of debugging the option of functioning of the

fuel and energy complex in the most loaded day;

- the stage of calculating and analyzing options for the inoperability of objects in the most loaded day.

At the stage of debugging the annual model variant, massive transformations of subject information are performed in order to obtain the target characteristics of the objects functioning. Produced here:

1. Debugging of the power and heat power unit, including the preparation of data for stations (or their groups) and boiler houses (or their groups), including: - transformation and verification of data on fuel consumption at the industry facilities (reduction to a single scale of measurement for the same coefficients for each resource);

- determination of the technological characteristics of the functioning of heat and power generating sources (volumes of heat and electricity supplied by them for certain types of fuel, specific fuel consumption);

- control over the correspondence of the supply of converted resources and fuel consumption inside the stations and boiler houses;

- control of the heat and electricity balance of throughout the country.

2. Debugging the fuel supply unit, including: - determination of the obligatory need for fuel by territories, if necessary, with a breakdown of the obligatory need by separate categories (for example, for the needs of the population and industry);

- data correction in case of imbalance in fuel consumption in the territories;

- analysis of the transport capacity infrastructure in the fuel industries.

3. Balance sheet estimate for all energy resources excluding their reserves.

4. Carrying out optimization calculations that determine:

- "locked" energy resources and determining the causes of their occurrence; - fuel shortages in the territories associated with the technological of the shortage energy transport infrastructure, or with an imbalance of their own production capabilities and needs in the event of an isolated territory;
- shortages of final types of energy resources, the reasons for their occurrence;

- the level of capacity utilization of production facilities in the fuel and energy complex.

At the stage of debugging the variant of the most loaded day, the coefficients of seasonal unevenness are determined, also the reserve capabilities of industries to cover additional needs for energy resources. At this stage, the following are carried out:

1. In the block of electric and heat power engineering

- correction the production capabilities of stations and boiler houses in the basic mode of their operation;

- determination of reserve equipment capabilities at thermal power plants and boiler houses;

- correction the demand for electricity and heat;

- verification of cost coefficients.

2. In the fuel supply unit:

- determination of the fuel industries reserves;

- correction of obligatory fuel need.

3. Debugging of the option, during which discrepancies are analyzed and eliminated in terms of fuel supply to consumers, its extraction from storage facilities, priority of fuel use in electric and heat power engineering.

At the stage of analyzing the consequences from outages of critical facilities, the relative changes in the analyzed indicators are determined for individual territories or their groups. This information is used to determine the CF for FEC.

\section{Method for determining of FEC critical facilities}

The method for determining the sectoral energy facilities criticality for territories in conditions of sectoral systems interconnected operation meets the following basic provisions:

1. The problem is solved within the framework of multivariate and multilevel computational experiments based on the fuel and energy complex models and its industries. A key feature of these experiments is the multiple use of the same data for different levels models. It is allowed to use the same information at different levels in a transformed form.

2. The task assumes specifying a list of industry critical objects, from which the fuel and energy complex critical facilities are selected. Scenarios of inoperability of industry elements are simulated in computational experiments.

3. The solution of the problem assumes a three-stage scheme for transforming the results of computational experiments, including obtaining quantitative, qualitative and expert assessments of the elements criticality. Qualitative and expert assessments are formed on the basis of quantitative ones. Qualitative assessments (categories of elements criticality) make it possible to single out groups of sectoral elements that are problematic to varying degrees for the fuel and energy complex as a whole. The main criterion is the integral criterion of the elements significance, which comprehensively characterizes the changes in the 
analyzed indicators in the event of industry elements inoperability.

4. The analyzed model indicators in relation to the task are systematized into two categories. The category of the most important indicators is formed by energy resource deficits, which are a system characteristic of the functioning of industries. The category of related indicators is represented by technologies for meeting the needs of the territories (reserves and reserves, the possibility of interchangeability of energy resources in technological processes). A correct analysis of the elements criticality requires consideration of both types of indicators.

The criterion for the significance of each element is determined for the entire set of calculate states with various combinations of its shutdowns:

$$
\begin{gathered}
Z O_{i}=\sum_{j=1}^{J}\left[\sum_{k=1}^{K} Z O_{i}^{j, k} \times Z C^{j}\right], \\
Z O_{i}^{j, k}=Z S_{r}^{j, k} /_{R},
\end{gathered}
$$

where

$Z O_{i}$ - the significance of the $i^{\text {th }}$ fuel and energy complex element,

$Z O_{i}^{j, k}$ - the significance of the $i^{\text {th }}$ element by the $j^{\text {th }}$ indicator in calculations with capacity outages groups $k$, $Z S_{r}^{j, k}$ - assessments of states for the $j^{\text {th }}$ indicator in calculations with the inclusion of the $i^{\text {th }}$ element in the capacity outage groups $k$,

$R$ - the number of states estimated by the $j^{\text {th }}$ indicator with the inclusion of the $i^{\text {th }}$ element in the capacity outage groups $k$,

$Z C^{j}$ - the significance (specific weight) of the $j^{\text {th }}$ indicator,

$K$ - maximum capacity of outage groups in optimization calculations,

$J$ - the number of analyzed indicators,

$I$ - a disconnected elements set of the fuel and energy complex, $i \in I$.

Formalization of the identification of the criticality category of elements (the first category is considered the most critical) can be represented as follows:

$$
\begin{gathered}
C A T_{i}^{W}=f\left(Z O_{i}^{w}, N\right), \\
N=\left\{n_{l}\right\}, l=\overline{1, L}, \\
n_{l}=\left\{n_{l}^{\text {low }}, n_{l}^{u p}\right\}, l=\overline{1, L}, \\
l,\left(Z T_{i}^{\text {max }} \times n_{l}^{\text {low }}\right) \leq Z O_{i}^{w} \leq\left(Z O_{i}^{\max } \times n_{l}^{\text {up }}\right) \\
1, Z O_{i}^{w} \geq\left(Z O_{i}^{\max } \times n_{l}^{\text {low }}\right), l=1 \\
Z O_{i}^{\text {max }}=\max \left(Z O_{i}^{w}\right), \mathrm{w}=\overline{1, W}
\end{gathered}
$$

where

$C A T_{i}^{w}$-the category of criticality for the $i^{\text {th }}$ element for the territory $w$,

$Z O_{i}^{w}$ - the coefficient of significance for the $i^{\text {th }}$ element for the territory $w$,

$Z O_{i}^{\max }$ - the maximum value of significance for the $i^{\text {th }}$ element for all considered territories,

$N$ - a set of specified criticality categories $n_{l}$ ( $L$ - the number of specified categories of elements criticality), $n_{l}^{\text {low }}, n_{l}^{\text {up }}$ - the lower and upper boundaries of the criticality category $l$,
$I-$ a set of disconnected elements of the fuel and energy complex,

$W$ - the number of considered territorial units.

Technically, the problem of determining the CF for the fuel and energy complex (fig. 2) is represented by the processes of data transformation and control during computational experiments, during the analysis of the calculated model indicators, and analysis of the industry elements criticality. The initial information of the task is represented by target settings (maximum power of outage groups, a list of analyzed industry elements and analyzed model indicators, indicators for a qualitative assessment) and a basic daily version of the model, the disturbed variant of which are calculated during of the experiments. The resulting information is quantitative, qualitative and expert assessments, on the basis of which the list of the FEC critical facilities is formed.

Formally, the method for determining the critical facilities for the fuel and energy complex is presented in three stages:

- stage of the strategy formation for choosing the fuel and energy complex critical facilities;

- stage of optimization calculations;

- the stage of forming the list of the fuel and energy complex critical facilities.

At the first stage:

- the objects of the modeled the fuel and energy complex structure are formed;

- groups of industry elements outage are formed for their inclusion in simulated situations;

- a set of criteria for assessing states (the most important and accompanying model indicators) is formed, the significance of these criteria is determined;

- the categories of elements criticality and their threshold values are determined.

At the second stage, optimization calculations of the fuel and energy complex model are carried out, within the framework of which emergency situations with failure of industry elements groups are calculated.

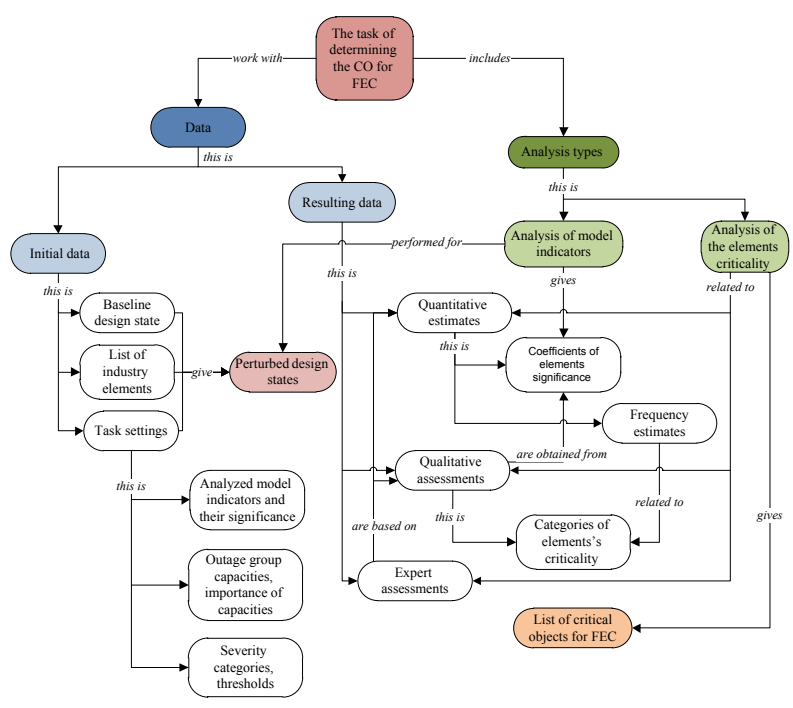

Fig. 2. Conceptual scheme for solving the problem of determining the $\mathrm{CF}$ for the fuel and energy complex. 
At the third stage, estimates of the disconnected industry elements criticality are formed with the formation of the fuel and energy complex critical facilities list:

- the composition of the analyzed calculated states is adjusted by excluding states with a relative deficit of at least one resource that is acceptable for the country as a whole (at this stage, a 5\% threshold for assessing the deficit is adopted);

- criteria for the elements significance for the territories under consideration or their groups are determined;

- the categories of criticality for the analyzed elements are determined;

- a list of the fuel and energy complex critical facilities is formed.

The algorithm of the forming the fuel and energy complex CF list is shown in fig. 3 .

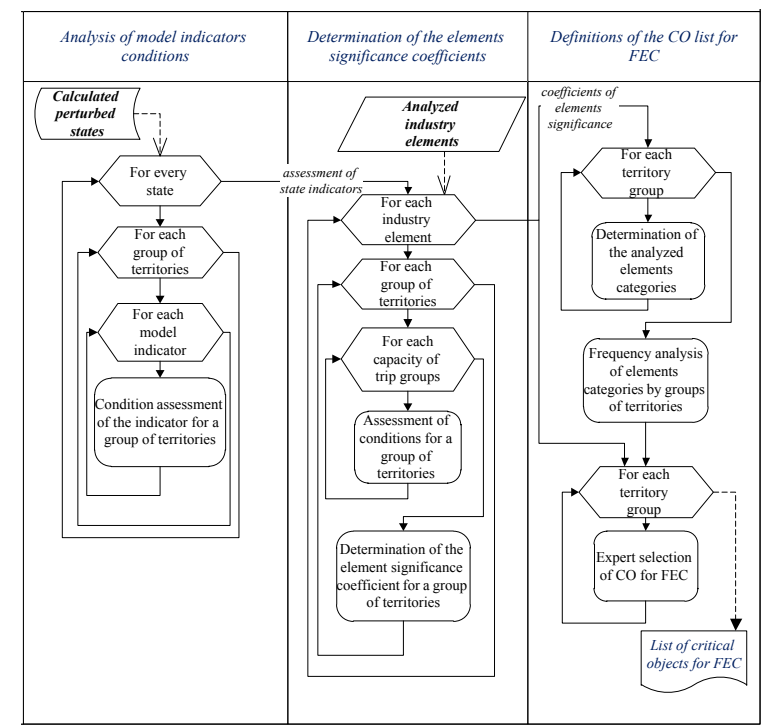

Fig. 3. Algorithm for the formation of the fuel and energy complex critical facilities list.

The presented method has been twice tested on fuel and energy complex models using the example of critical facilities shutdowns in the gas industry. According to the results of the first test approbation, the efficiency and effectiveness of the developed method were proved, some of its key points were adapted [7]. The current approbation of the method was carried out on a more flexible and more adequate version of the model, taking into account the reserve supply of electricity and heat from the electric and heat power stations. The analysis of these results was aimed at:

- identifying disagreements in the priority of the gas industry critical objects and the fuel and energy complex objects;

- assessment of the plausibility of the method, taking into account the influence of the fuel and energy complex systemic effect.

\section{Conclusion}

The paper presents the continuity of the method for choosing the fuel and energy complex critical facilities. A general scheme for the selection of the fuel and energy complex critical facilities on the basis of industry critical elements has been developed and formalized. A comparative characteristic of the sectoral (on the example of the gas industry) method for the selection of critical elements and the method for the formation of the critical facilities list in the fuel and energy complex taking into account the systemic effect is given.

The article formalizes the territorial-production model of the fuel and energy complex, which use to determine the fuel and energy complex critical facilities, describes the implemented three-stage scheme of working with it. The developed method for determining the fuel and energy complex critical facilities in the conditions of the interconnected operation of industry systems is presented. For the selection of the fuel and energy complex critical facilities the most important and accompanying model indicators are highlighted, an integral indicator of the industry elements criticality is proposed.

The studies presented in this work were carried out with the financial support of the RFBR grant No. 20-0800367.

\section{References}

1. H. Su, E. Zio, J. Zhang, X. Li. A systematic framework of vulnerability analysis of a natural gas pipeline network. Reliability Engineering \& System Safety, 175, 79-91(2018)

2. R. Carvalho, L. Buzna, F. Bono, M. Masera, D.K. Arrowsmith, D. Helbing. Resilience of natural gas networks during conflicts, crises and disruptions. PloS one, 9(3) (2014)

3. N. Pyatkova, N. Beresneva, A. Edelev. Research of critical energy infrastructures taking into account energy security. Methodological Problems in Reliability Study of Large Energy Systems (RSES 2017), 5 (2017)

4. S.M.Senderov, S.V.Vorobev. Approaches to the identification of critical facilities and critical combinations of facilities in the gas industry in terms of its operability. Reliability Engineering \& System Safety, 203 (2020).

5. S.V. Vorobiev, A.V. Edelev. Search for critical objects of the gas industry using the method of determining critical elements in the networks of technical infrastructures. Methodological issues of researching the reliability of large energy systems, 68, 76-84 (2017).

6. N.I. Pyatkova, S.M. Senderov, E.V. Pyatkova. Methodological features of the study of energy security problems at the present stage. Izvestiya RAN. Energy, 2, 81-87 (2014)

7. N.M. Beresneva, N.I. Pyatkova. The approach to determining vulnerable elements in critical energy infrastructures. Rudenko International Conference "Methodological problems in reliability study of large energy systems" (RSES 2019), 139 (2019). 